\title{
Single sideband FBMC system for 2-km SMF transmission
}

\author{
Tingting Zhang ${ }^{1}$, Mary Mccarthy ${ }^{1}$, Stylianos Sygletos ${ }^{1}$, Filipe Ferreira ${ }^{1}$, Andrew D. Ellis ${ }^{1}$ \\ ${ }^{1}$ Aston Institute of Photonic Technologies (AIPT), Aston University, Birmingham, B4 7ET, UK \\ E-mail address: andrew.ellis@aston.ac.uk
}

\begin{abstract}
We report a 144-Gb/s single sideband filter bank multicarrier system supporting transmission of studio quality ultra-high definition TV over 2-km single mode fiber (SMF) and the importance of optimizing the modulation index is highlighted.

OCIS codes: (060.2330) Fiber optics communications; (060.2360) Fiber optics links and subsystems.
\end{abstract}

\section{Introduction}

The spread of bandwidth-hungry services such as broadband mobile communication, high-definition television streaming and cloud computing have resulted in increasing demand for high-capacity short reach optical networks. Because large amount of transceiver unites are used in short-haul optical links, power consumption, footprint and cost efficiency are three main factors for short reach optical links design, biasing designs towards direct detection even for data rates beyond $100 \mathrm{~Gb} / \mathrm{s}$. This is especially the case for ultra-high definition television, where uncompressed data rates peak at $144 \mathrm{~Gb} / \mathrm{s}$ and high volume use is foreseeable. With the help of polarization multiplexing, dual polarization $100 \mathrm{~Gb} / \mathrm{s}$ per lane has been demonstrated by utilizing 4-level pulse amplitude modulation (PAM4) [1] or half-cycle Nyquist subcarrier modulation [2]. However, complex polarization tracking, polarization multiplexing and de-multiplexing are required, which diminish the inherent benefits of direct detection. Recent research interest in the capacity of short reach optical networks has moved towards $100 \mathrm{~Gb} / \mathrm{s}$ single polarization per lane, which can be realized by utilizing advanced modulation formats such as discrete multi-tone [3], multi-band carrier-less amplitude and phase modulation [4] and PAM4 [5]. In such systems, complex digital signal processing (DSP) is required to address chromatic dispersion or bandwidth effects, resulting in high power consumption. A recently proposed allanalogue filter bank multicarrier (FBMC) technique based on mature and stable electrical and microwave components provides a promising solution for DSP-less broadband optical links [6]. Compared with conventional subcarrier multiplexing, FBMC has higher spectral density thanks to the orthogonality of the overlapping sub-channels and no cyclic prefix is required in comparison with orthogonal-frequency-division-multiplexing. However, to date, the per lane data rate of FBMC system is only $16.2 \mathrm{~Gb} / \mathrm{s}$ [6].

In this paper, a high-capacity single sideband (SSB) FBMC system using direct detection is proposed and numerically investigated. To achieve transmission data rate as high as $144 \mathrm{~Gb} / \mathrm{s}$ with an electro-optical bandwidth of less than $40 \mathrm{GHz}, 16$ quadrature amplitude modulation (16QAM) is used as the modulation format. To minimize composite second order (CSO) distortion in the photodetector (PD), the IQ modulator is biased at quadrature. The signal to noise ratio (SNR) and inter subcarrier interference may be optimized through the number of subcarriers and the modulation index. For a fixed number of subcarriers in optical back-to-back case, the optimum modulation index depends on the received optical power, the crosstalk induced by square-law photo-detection, and to a lesser extent the nonlinearity of IQ modulator. For system reach of 2-km SMF, using more subcarriers with an optimized modulation index enables the system to achieve better transmission performance due to enhanced dispersion tolerance.

\section{Simulation Setup}

Figure 1 shows the schematic diagram of the proposed SSB FBMC system. In our simulation, the 16QAM signal for each sub-channel was generated from fully random binary sequences which were Gray coding and mapped into 16QAM before being up-sampled to 256 samples per symbol. Both of the in-phase and quadrature information of the generated 16QAM signal were bipolar 4 amplitude shift keying modulated, which were then modulated to different RF subcarriers, with subcarrier frequency spacing equal to the symbol rate and relative phases of zero, by utilizing ideal IQ mixers. A laser with the wavelength of $1550 \mathrm{~nm}$ and a linewidth of $100 \mathrm{kHz}$ was used as the optical source and the maximum output power was $10 \mathrm{dBm}$. To generate an optical SSB signal, the multiplexed electrical signal was fed to one port of the IQ modulator with 3-dB insertion loss and no bandwidth limitation, whilst its Hilbert transform (obtained with a $90^{\circ}$ phase shifter) was sent to the other port with the optical phase shift between two sub modulators also set to be $90^{\circ}$. Both two sub modulators were biased at quadrature resulting in a strong optical carrier whilst simultaneously minimizing CSO distortion. The receiver consisted of a $50-\mathrm{GHz}$ photodetector with responsitivity of $0.85 \mathrm{~A} / \mathrm{W}$, shot noise and thermal noise $(18 \mathrm{pA} / \sqrt{\mathrm{Hz}})$, and a set of IQ mixers for down-conversion. To emulate RF carrier recovery, the down-converted in-phase and quadrature signals of each sub-channel were considered as a single 16QAM signal. Since temporally rectangular pulses were used in the transmitter, a Sinc-shaped 
filter matched at the symbol rate and truncated at $144 \mathrm{GHz}$ was employed for each sub-channel in the receiver. The down-sampling position was obtained by calculating the minimum variance of the received signal. Then a steepest descent method [7] was utilized to compensate for the constellation rotation after down-sampling to 1 sample per subcarrier symbol. Finally, the BER was calculated using error counting of the demodulated signal. The length of the transmitted bit stream was calculated to be at least 100 times than the inverse of the desired BER, which was initially estimated with a trial of 1048576 bits.

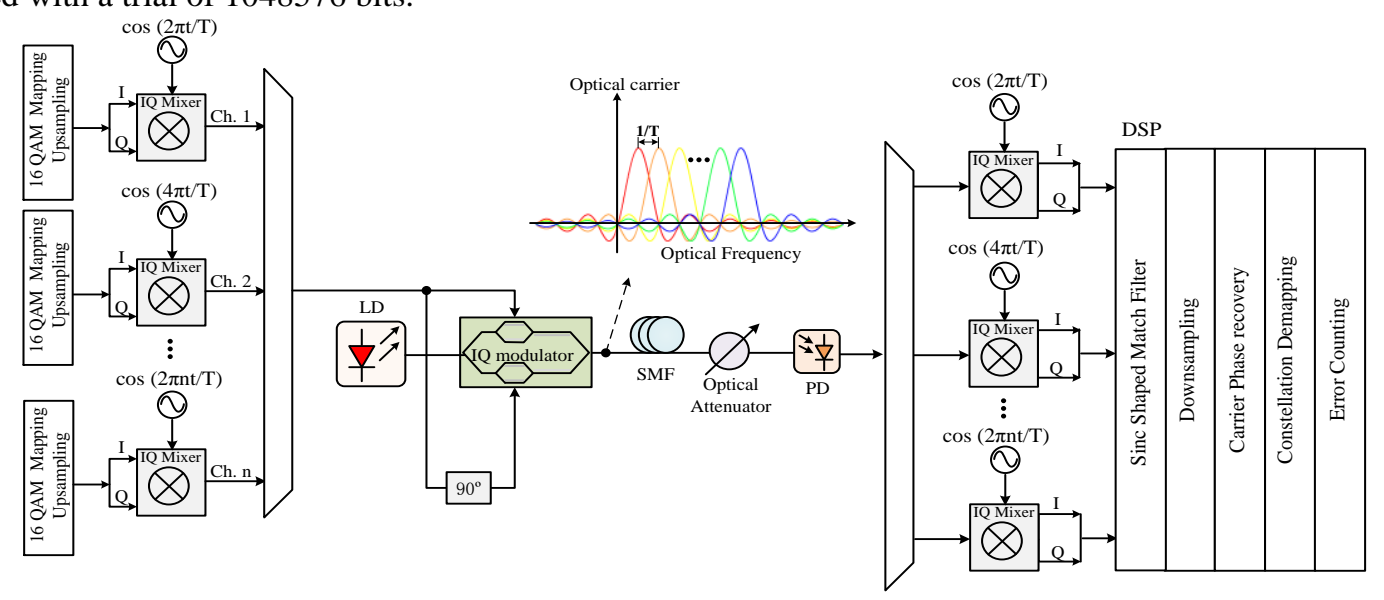

Fig. 1 The schematic diagram of the proposed SSB FBMC system.

\section{Results and Discussion}

To illustrate the definition of the modulation index used in this paper, the subcarrier multiplexed signal fed to the upper arm of the IQ modulator can be written as $S_{\mathrm{up}}(t)=\operatorname{Real}\left(\sum_{k=1}^{N_{\mathrm{c}}} \beta * u_{k}(t) \exp \left(\mathrm{j} \omega_{k} t\right)\right)$, where $\mathrm{N}_{\mathrm{c}}$ is the number of subcarriers, $\beta$ is the modulation index for each sub-channel and is same for all the sub-channels, $u_{k}(t)$ is 16QAM modulated information for the $k^{\text {th }}$ subcarrier with any value of $\pm 1 \pm i, \pm 1 \pm 3 i, \pm 3 \pm i$, and $\pm 3 \pm 3 i, \omega_{k}$ is the angular frequency for the $\mathrm{k}^{\text {th }}$ subcarrier and is equal to $2 \pi k / \mathrm{T}$, where $\mathrm{T}$ is the symbol time. Figure 2 (a) shows the BER performance at a fixed received optical power of $-8 \mathrm{dBm}$, as the modulation index $\beta$ is varied for a range of different number of subcarriers. The lowest BER for different number of subcarriers all show optimum performance when $\mathrm{N}_{\mathrm{c}} * \beta^{2}$ is about 0.008 and the optimum point is approximately independent of the number of subcarriers. This represents a fixed optical power level in the data sideband and is proportional to the rms drive signal power.

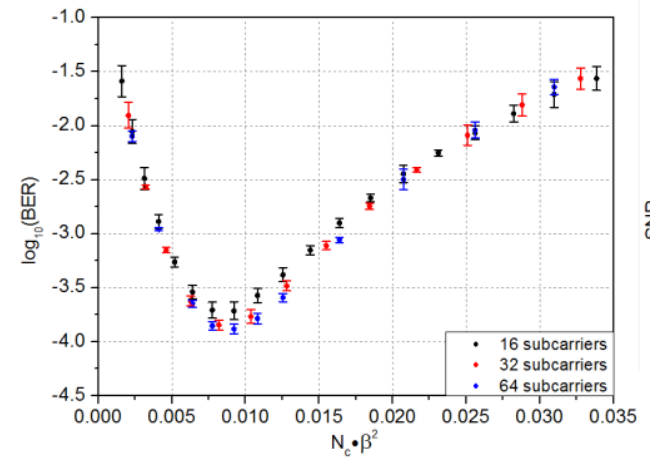

(a)

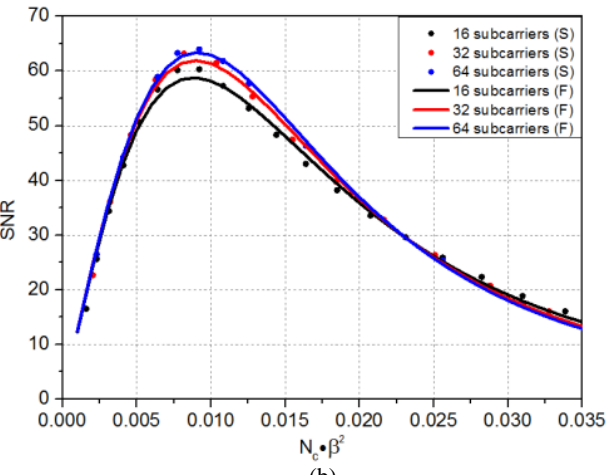

(b)

Fig. 2 (a) BER performance of the proposed FBMC system for different numbers of subcarriers at the received optical power of $-8 \mathrm{dBm}$. (b) SNR at the received optical power of $-8 \mathrm{dBm}$. Dot: simulation results. Straight line: fitting curve.

To get a better understanding of this phenomenon, the SNR at the received optical power of $-8 \mathrm{dBm}$ can be obtained using formula (4) in Ref. [8]. Figure 2 (b) shows the calculated SNR (dots) and corresponding curve fits considering thermal noise, shot noise, CSO distortion generated in PD and modulator nonlinearity by using a custom formula with the form of $s n r=x /\left(\mathrm{a}+\mathrm{b} x^{2}+\mathrm{c} x^{4}\right)$ in Matlab. Here, $x$ is equal to $\mathrm{N}_{\mathrm{c}} * \beta^{2}$ and is proportional to the power of the desired electrical signal. Coefficient a is related to shot noise and thermal noise, which is approximately the same in all cases, dominating the performance at low signal power $(x<0.005)$. Coefficient $\mathrm{b}$ and $\mathrm{c}$ are related to CSO distortion in the PD and composite triple beat distortion caused by the modulator respectively. At larger signal 
power, the SNR degrades firstly due to the increase of CSO distortion in the PD, which is smaller for large number of subcarriers due to spread of the signal power between subcarriers, and secondly due to increasing distortion from the modulator, which increases with the number of subcarriers because of the increasing mixing products. At optimum signal power, distortion from the modulator is small and can be ignored compared with distortion from the photodiode. Since receiver noise is same regardless of the number of subcarriers and the power of CSO distortion located within the desired signal bandwidth is smaller when more subcarriers are used, slightly higher SNR may be achieved in systems using more subcarriers.

Since the optimum modulation index depends on the tradeoff between the linear SNR and nonlinear distortion in the photodiode, it is critically dependent on the received optical power. This can be seen from the fitting formula which has a shot and thermal noise related coefficient a. Therefore, the optimum modulation index ensuring minimal BER should be adjusted respectively according to the received optical power when comparing different system deployments, including transmission over the maximum reach considered here, 2-km SMF. Contour plots in Fig. 3 show that after 2-km transmission, the minimum BER of 16-subcarrier system is only about $10^{-4}$ at the available maximum received optical power of $6.6 \mathrm{dBm}$, while at much lower received optical power of $0.6 \mathrm{dBm}$, BER of $10^{-6}$ is realized in the 64-subcarrier system. Transmission penalties are dominated by the higher frequency subcarriers and the penalties associated with the 16-subcarrier system are attributed to chromatic dispersion, which we believe reduces the inter subcarrier orthogonality.

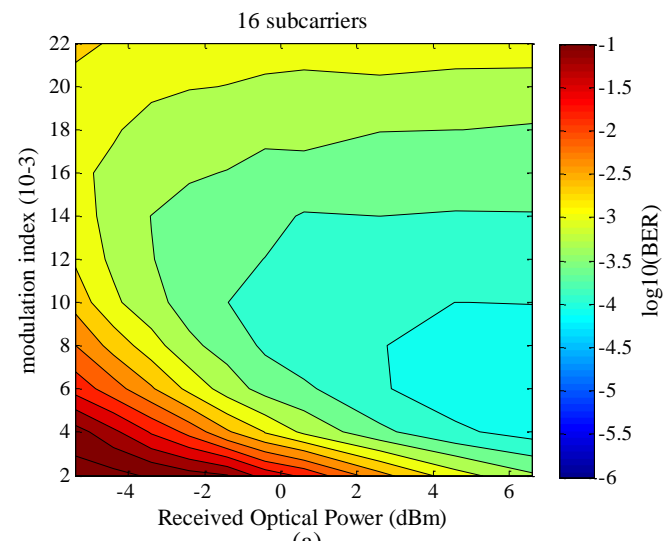

(a)

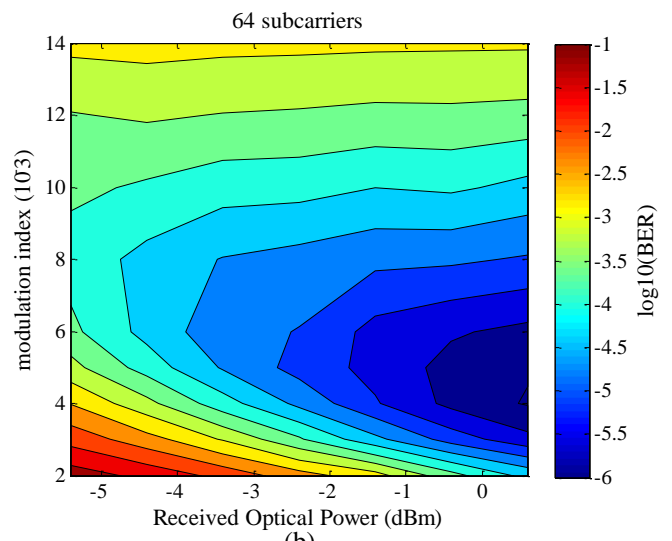

(b)

Fig. 3 Contour plots of BER performance of (a) 16-subcarrier and (b) 64-subcarrier FBMC system.

\section{Conclusion}

The performance of the proposed single sideband FBMC system based on direct detection using different number of subcarriers has been studied respectively for optical back-to-back and 2-km transmission. To avoid modulator distortion, a small modulation index is always preferred and the optimum value depends on both receiver noise and CSO distortion. For 2-km transmission, better system performance can be achieved by using more subcarriers.

\section{Acknowledgements}

This work was supported in part by the EPSRC through projects EP/L00044X/1 and EP/L000091/1(Silicon Photonics for Future Systems and PEACE).

\section{References}

[1] R. Rodes, J. Estaran, B. Li, M. Muller, J. B. Jensen, T. Gruendl, M. Ortsiefer, C. Neumeyr, J. Rosskopf, K. J. Larsen, M. C. Amann, and I. T. Monroy, "100 Gb/s single VCSEL data transmission link," in Optical Fiber Communication Conference (OFC), 2012, PDP5D.I0.

[2] A. S. Karar and J. C. Cartledge, "Generation and Detection of a 112-Gb/s Dual Polarization Signal Using a Directly Modulated Laser and Half-Cycle 16-QAM Nyquist-Subcarrier-Modulation," in European Conference and Exhibition on Optical Communication, 2012, Th.3.A.4.

[3] T. Takahara, T. Tanaka, M. Nishihara, Y. Kai, L. Li, Z. Tao, and J. Rasmussen, "Discrete Multi-Tone for 100 Gb/s Optical Access Networks," in Optical Fiber Communication Conference (OFC), 2014, M2I.1.

[4] M. I. Olmedo, T. J. Zuo, J. B. Jensen, Q. W. Zhong, X. G. Xu, S. Popov, and I. T. Monroy, "Multiband Carrierless Amplitude Phase Modulation for High Capacity Optical Data Links," J. Lightw. Technol., 32, 798-804 (2014).

[5] K. P. Zhong, X. Zhou, Y. L. Gao, W. Chen, J.W. Man, L. Zeng, A. P. T. Lau, and C. Lu, "140-Gb/s 20-km Transmission of PAM-4 Signal at $1.3 \mu \mathrm{m}$ for Short Reach Communications," IEEE Photon. Technol. Lett., 27, 1757-1760 (2015).

[6] F. A. Gutiérrez, P. Perry, E. P. Martin, A. D. Ellis, F. Smyth and L. P. Barry, "All-Analogue Real-Time Broadband Filter Bank Multicarrier Optical Communications System," J. Lightw. Technol., 33, 5073-5083 (2015).

[7] Irshaad Fatadin, David Ives, and Seb J. Savory, "Blind Equalization and Carrier Phase Recovery in a 16-QAM Optical Coherent System," J. Lightw. Technol., 27, 3042-3049 (2009).

[8] R. A. Shafik, M. S. Rahman and A. R. Islam, "On the Extended Relationships Among EVM, BER and SNR as Performance Metrics," in International Conference on Electrical and Computer Engineering, 2006, pp. 408-411. 